\title{
Defect Chaos and Bursts: Hexagonal Rotating Convection and the Complex Ginzburg-Landau Equation
}

\author{
Santiago Madruga, ${ }^{1}$ Hermann Riecke, ${ }^{1}$ and Werner Pesch ${ }^{2}$ \\ ${ }^{1}$ Engineering Science and Applied Mathematics, Northwestern University, Evanston, Illinois 60208, USA \\ ${ }^{2}$ Physikalisches Institut, Universität Bayreuth, D-95440 Bayreuth, Germany
}

(Received 27 July 2005; revised manuscript received 12 October 2005; published 22 February 2006)

\begin{abstract}
We employ numerical computations of the full Navier-Stokes equations to investigate non-Boussinesq convection in a rotating system using water as the working fluid. We identify two regimes. For weak nonBoussinesq effects the Hopf bifurcation from steady to oscillating (whirling) hexagons is supercritical and typical states exhibit defect chaos that is systematically described by the cubic complex Ginzburg-Landau equation. For stronger non-Boussinesq effects the Hopf bifurcation becomes subcritical and the oscillations exhibit localized chaotic bursting, which is modeled by a quintic complex Ginzburg-Landau equation.
\end{abstract}

DOI: 10.1103/PhysRevLett.96.074501

The complex Ginzburg-Landau equation (CGL) as the universal description of weakly nonlinear oscillations has been studied theoretically in great detail. The classical, supercritical case, which involves only two independent parameters, looks deceptively simple. It exhibits, however, a vast variety of qualitatively different phenomena including several types of spatiotemporal chaos [e.g., [1,2]]. If the oscillations arise in a subcritical Hopf bifurcation the quintic CGL comes into play, which introduces further interesting states including, for instance, intermittent bursts in the oscillation amplitude.

In contrast to the extensive theoretical work on the CGL, direct experimental validation of its various regimes of complex behavior are scarce [3], in particular, for the two-dimensional case [4]. In this Letter we present detailed numerical computations for an experimentally realizable thermal convection experiment that exhibit defect chaos and bursts. We show that the defect chaos can be systematically described by a cubic CGL, while the bursts can be modeled with a quintic one.

Rayleigh-Bénard convection of a fluid layer heated from below in systems with large aspect ratio, in which the lateral dimension $L$ of the layer is much larger than its thickness $h$, has proved to be a paradigmatic experimental system [5] for studies of complex patterns. Above a critical temperature difference $\Delta T_{c}$ across the layer, which corresponds to the critical value $R_{c}$ of the dimensionless Rayleigh number $R$, one observes in the simplest case the familiar striped (roll) patterns with wave numbers $q$ close to the critical wave number $q_{c}$. However, in systems in which $\Delta T_{c}$ is large, fluid properties like the thermal expansion coefficient or the viscosity vary significantly across the layer. Under these non-Boussinesq (NB) conditions the instability of the homogeneous state leads to hexagonal convection patterns [6].

If the chiral symmetry of the system is broken by rotating the layer about a vertical axis with frequency $\Omega$, new interesting dynamics arise. For $\Omega$ above the Küppers-Lortz
PACS numbers: 47.52.+j, 05.45.Jn, 47.20.Lz, 47.27.T-

frequency $\Omega_{\mathrm{KL}}$, one finds in the Boussinesq case immediately at onset $R_{c}$ domain chaos in which rolls persistently switch orientation [cf. [5] ]. In contrast, in the NB case the hexagons are steady in this regime. However, weakly nonlinear theory predicts at $\epsilon \equiv\left(R-R_{c}\right) / R_{c}=\epsilon_{H}$ a secondary oscillatory instability [cf. Fig. 1] to "whirling hexagons," in which the three hexagon amplitudes oscillate about their mean, with their phases shifted by $2 \pi / 3$ relative to each other, giving the hexagons the appearance of rotating American footballs [7-9].

Here we perform computer experiments on rotating nonBoussinesq convection with water as the working fluid in cases that can be realized in currently available experiments. Focusing on the whirling hexagons, we obtain different spatiotemporally disordered states, an example of which is shown in Fig. 2(a), and use the complex oscillation amplitude $\mathcal{H}$ [see (2) below] to interpret them in terms of suitable complex Ginzburg-Landau equations. The magnitude $|\mathcal{H}|$ is shown in Fig. 2(b).

Specifically, we consider two situations corresponding to weak and intermediate NB effects, respectively, and solve the full Navier-Stokes (NS) equations with the leading-order temperature dependence of all fluid parameters included [10]. We obtain spatially periodic stationary hexagon patterns and test their stability using a standard Galerkin method. The temporal evolution of the states is

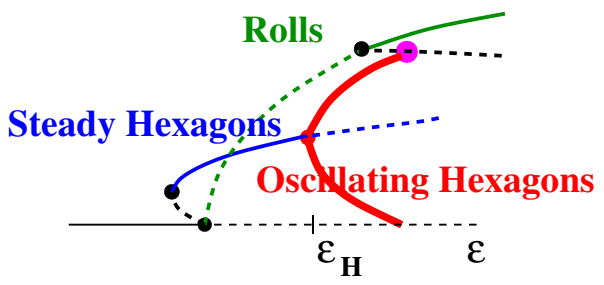

FIG. 1 (color online). Sketch of bifurcation diagram for rotating NB convection. Solid (dashed) lines denote stable (unstable) branches. For the oscillating ("whirling") hexagons maxima and minima of the oscillations are indicated (thick solid lines). 
then simulated with a pseudospectral code with periodic lateral boundary conditions $[10,11]$. We consider two setups differing in the mean temperature $T_{0}$ of the fluid layer. In case $A$ we choose $T_{0}=14{ }^{\circ} \mathrm{C}$ resulting in $\Delta T_{c}=6.4{ }^{\circ} \mathrm{C}$ and a value of $Q=-2.2$ for Busse's NB parameter [6]; case $B$ with $T_{0}=12{ }^{\circ} \mathrm{C}$ and $\Delta T_{c}=8.3^{\circ} \mathrm{C}$ is more nonBoussinesq and yields $Q=-3.62$ [12]. In both cases $h=$ $0.492 \mathrm{~cm}$ and $\Omega=65 \nu_{0} / h^{2}$ with $\nu_{0}$ being the viscosity of the fluid in the midplane.

In case $A$ we obtain above the Hopf bifurcation at $\epsilon_{H}$ states in which almost all convection cells are oscillating about a mean, but for general initial conditions the oscillations of different cells are out of phase with respect to each other. This is shown in Fig. 2(a) where the solution (with $N=256$ ) is visualized by the deviation $\theta(\mathbf{r}, t)$ of the temperature from the conduction profile in the midplane of the fluid layer. Here $\mathbf{r}$ denotes the horizontal coordinates. To extract the oscillations explicitly we make use of the fact that the underlying hexagon pattern itself is well ordered. We first demodulate each snapshot in space by writing

$$
\theta(\mathbf{r}, t)=\sum_{j=1}^{3} A_{j}(\mathbf{r}, t) e^{i q_{c} \hat{\boldsymbol{n}}_{j} \cdot \mathbf{r}}+\text { c.c. }+ \text { h.o.t. }
$$

Here the wave vectors $q_{c} \hat{\boldsymbol{n}}_{j}$ represent the three dominant wave vectors of the hexagons and h.o.t. denotes their harmonics. For strictly periodic stationary hexagon patterns the amplitudes $A_{j}, j=1 \ldots 3$, do not depend on $\mathbf{r}$ or $t$, have the same modulus $A_{\text {hex }}$, and can be chosen real and positive in our case (water). In general, the amplitudes $A_{j}(\mathbf{r}, t)$ are complex and depend slowly on space representing the contributions from the sidebands of the basic wave vectors $q_{c} \hat{\boldsymbol{n}}_{j}$.

Slightly above the Hopf bifurcation at $\epsilon=\epsilon_{H}$ the amplitudes $A_{j}$ vary in time. For patterns that are close to periodic in space the $A_{j}$ can be expressed as

$$
\begin{aligned}
A_{j}= & \left(A_{\text {hex }}+\mu^{1 / 2}\left[e^{j 2 \pi i / 3} \mathcal{H} e^{i \omega t}+\text { c.c. }\right]\right. \\
& +\mathcal{O}(\mu)) \exp \left(i \Delta q \hat{\boldsymbol{n}}_{j} \cdot \mathbf{r}+i \mu^{1 / 2} \phi_{j}(\mathbf{r}, t)\right),
\end{aligned}
$$

(a)

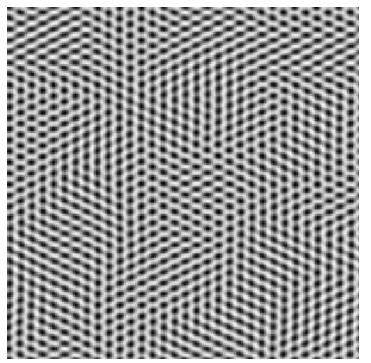

(b)

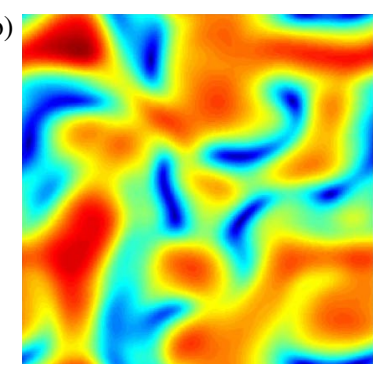

FIG. 2 (color online). Disordered state of whirling hexagons in case $A$ for $\epsilon=0.2 \quad\left(R_{c}=6141.3, \epsilon_{H}=0.062\right), \quad L=$ $32\left(2 \pi / q_{c}\right)=38.3$. (a) Snapshot of $\theta(\mathbf{r})$ [see Eq. (2)]. (b) Magnitude $|\mathcal{H}|$ of oscillation amplitude of snapshot shown in (a). Red (blue) indicates large (small) values of $|\mathcal{H}|$. where $\mu=\left(R-R_{H}\right) / R_{H}$ and $\omega$ is the Hopf frequency. Here $\mathcal{H}$ denotes the complex oscillation amplitude, which we extract by standard spatiotemporal demodulation of a series of snapshots like Fig. 2(a). The space dependence of the phases $\phi_{j}$ captures slight deformations of the underlying hexagon lattice.

A snapshot of the magnitude $|\mathcal{H}|$ of the oscillation amplitude is shown in Fig. 2(b). A more detailed analysis shows that the domains with small $|\mathcal{H}|$ are due to spiral defects in the complex oscillation amplitude, which are isolated zeroes of $\mathcal{H}$. During the evolution of the system these defects are persistently created and annihilated in pairs. In appearance, this state is thus quite similar to the defect chaos of the cubic CGL (see below).

When the NB effects are stronger (case $B$ ) the Hopf bifurcation is shifted to larger values of $\epsilon$ and, unexpectedly, becomes subcritical. The numerically determined bifurcation diagram showing the jump in the oscillation amplitude, the small hysteresis, and the restabilization [reentrance [13]] of the steady hexagons is presented in Fig. 3(a). As a consequence, the dynamical states obtained in this regime are very different. This is shown in Fig. 3(b), where the colors represent the oscillation magnitude [cf. Fig. 2(b)]. In addition, the contour lines indicate the underlying hexagon pattern. Strikingly, the oscillations are now localized into relatively small domains. Only within these domains the convection cells are elongated to footballs and are whirling. In most parts of the system the hexagons are relatively steady. The burstlike temporal evolution of the hexagon amplitudes is evident from Fig. 4. It shows the normalized oscillation intensity in the bursts $I(t)=$ $\mathcal{N}^{-1} \int_{|\mathcal{H}|>0.5|\mathcal{H}|_{\max }}|\mathcal{H}| d x d y$, where $\mathcal{N}$ is the temporal mean of the integral, which exhibits substantial intermittency reflecting the growth and decay of bursts (thick solid line). In case $A$ (thin solid line) the intensity fluctuates relatively little.

To provide insight into the complex dynamics obtained in our numerical experiments we make use of the fact that the whirling hexagons arise from a Hopf bifurcation. It is to be expected that in the supercritical case the dynamics can

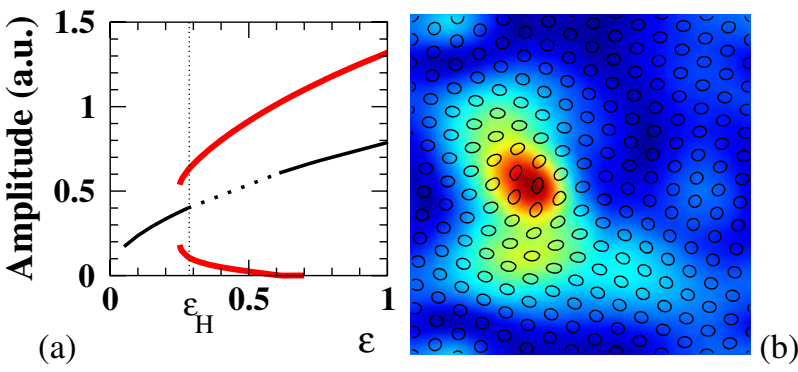

FIG. 3 (color online). (a) Bifurcation diagram for the subcritical case $B$ (cf. Fig. 1 with the rolls omitted). (b) Bursting state obtained with NS simulation $(N=128)$ in case $B$ for $\epsilon=0.5$ $\left(R_{c}=6121.9, \epsilon_{H}=0.285\right)$ and $L=16\left(2 \pi / q_{c}\right)=19.1 .|\mathcal{H}|$ color coded as in Fig. 2(b); contour lines indicate hexagon pattern. 


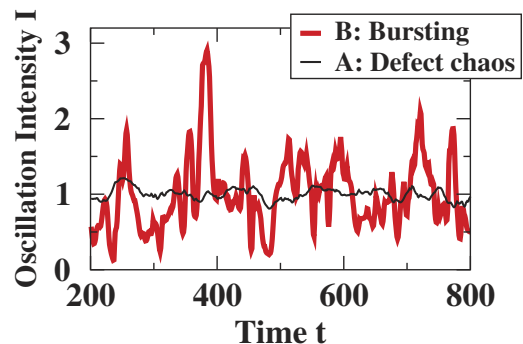

FIG. 4 (color online). Normalized intensity $I(t)$ (see text).

be captured by the CGL for the oscillation amplitude,

$$
\partial_{T} \mathcal{H}=\mu \mathcal{H}+d \nabla^{2} \mathcal{H}-c \mathcal{H}|\mathcal{H}|^{2} .
$$

We compute $d \equiv d_{r}+i d_{i}$ from the wave number dependence of the Floquet exponent of the Galerkin stability computations and extract $c \equiv c_{r}+i c_{i}$ from fits of (3) to the magnitude and frequency of growing oscillations obtained in direct simulations of the NS equations. Using a resolution corresponding to $N=256$ in Fig. 2, we find $d=$ $244+9.57 i$ and $c=219+1737 i$. Going to $N=512$ changes $c$ by less than $1 \%$. Consistent with predictions based on a theory for small-amplitude hexagons [9], these values position the system in the Benjamin-Feir-stable regime $\left(1+d_{i} c_{i} / d_{r} c_{r}>0\right)$ in which, however, defect chaos persists due to the wave number selection by the spiral defects $[1,2]$. Indeed, we find in the NS simulations that spatially homogeneous oscillations are stable. To reach the chaotic attractor shown in Fig. 2 we employ initial conditions in which the oscillations are not synchronized across the system.

To capture the bursting dynamics found in the subcritical case $B$ the CGL (3) has to be extended to include a quintic term, $-g|\mathcal{H}|^{4} \mathcal{H}$. Proceeding as in case $A$, we determine the coefficients of the resulting quintic CGL and simulate it. As shown in Fig. 5(a), which depicts a snapshot of the magnitude $|\mathcal{H}|$, we obtain quite similar bursts. Based on this qualitative agreement we use the CGL to gain further insight into the bursting found in the NS simulations.

The burst mechanism can be elucidated by considering the local gradient of the phase of $\mathcal{H} \equiv \mathcal{R} e^{i \psi}$, i.e., the wave vector $\mathbf{k} \equiv \nabla \psi$ of the oscillations. Note that $\mathbf{k}$ is not related to the wave vectors making up the hexagon pattern. Gradients in $|\mathcal{H}|$ induce significant differential phase winding due to the strong amplitude dependence of the oscillation frequency $\left(\left|c_{i} / c_{r}\right| \gg 1\right)$. This builds up $|\mathbf{k}|$ at the perimeter of the bursts while $|\mathbf{k}|$ remains small at their cores, as illustrated in Fig. 5(b), which shows an enlargement of a burst (top) and the associated $|\mathbf{k}|$ (bottom). The increased wave number $\mathbf{k}$ enhances the dissipation via the diffusion term in (3) and eventually leads to a collapse of the oscillation amplitude. This mechanism has been studied previously in one dimension [14-19]. It has been shown to underlie the dispersive chaos observed in binarymixture convection $[15,16]$ and, interestingly, can be strong enough to avoid blowup even if there is no saturat- (a)

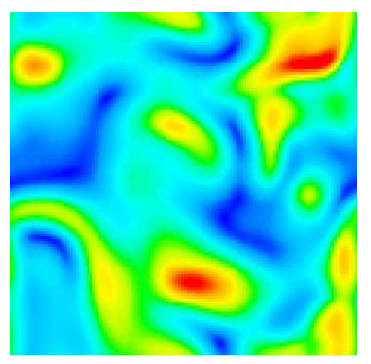

(b)

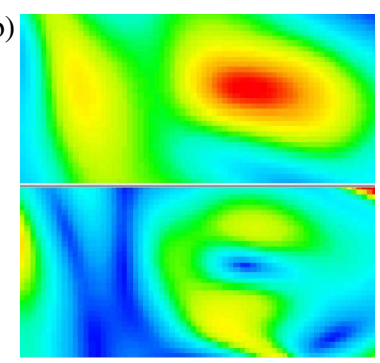

FIG. 5 (color online). Bursting state obtained with quintic CGL for parameters corresponding to Fig. 3, $d=22.4+$ $0.389 i, c=-12.96+84.70 i, g=42.53+17.20 i$, except for the larger system size, $L=100$. (a) Magnitude $|\mathcal{H}|$ of oscillation, (b) enlarged portion from (a) magnitude $|\mathcal{H}|$ (top) and wave number $|\mathbf{k}| \equiv|\nabla \arg (\mathcal{H})|$ of the oscillation (bottom).

ing nonlinear term at all $\left(c_{r}=g_{r}=0\right)[14,17,18]$. The collapse can also be interpreted in terms of two colliding fronts that connect the steady base state with the nonlinear oscillatory state. Since these fronts select a nonvanishing wave number for the nonlinear state, the base state can invade the nonlinear state even above the Hopf bifurcation ("retracting fronts") [19]. Note that the collapse of the bursts described here is not due to a breakdown of the underlying hexagonal structure [20,21].

Further analysis of the NS simulations shows that the localization mechanism has an additional contribution. Since the oscillations arise in a secondary bifurcation they couple also to slow, long-wave deformations of the hexagon lattice, which are described by an additional, diffusive equation for the phase vector $\overrightarrow{\boldsymbol{\phi}} \equiv\left[\phi_{1},\left(\phi_{2}-\phi_{3}\right) / \sqrt{3}\right]$ [9]. Thus, deformations of the hexagon lattice affect the oscillations through an additional term $\sim \mathcal{H} \nabla \cdot \overrightarrow{\boldsymbol{\phi}}$ to be included in (3), which captures the dependence of the growth rate $\sigma$ of the oscillations on local compressions of the lattice. Conversely, gradients in $\mathcal{H}$ drive $\overrightarrow{\boldsymbol{\phi}}$ [9]. Figure 6(a) shows that the hexagonal lattice is dilated inside the burst $(\nabla \cdot \overrightarrow{\boldsymbol{\phi}}<0)$. This decrease in the local wave number $q \equiv q_{c}+\nabla \cdot \overrightarrow{\boldsymbol{\phi}} / 2$ of the hexagons increases $\sigma$ [see Fig. 6(b)] and provides a positive feedback for the oscillations that enhances the bursting activity compared to the quintic CGL.

Of course, the coupling of the oscillations to the underlying lattice exists also in the supercritical case shown in Fig. 2. Consistent with the weakly nonlinear description of rotating hexagons [9], we find, however, that in this regime the growth rate $\sigma$ depends only weakly on the local wave number of the hexagons [see Fig. 6(b)] and varies by less than $10 \%$ across the system. We therefore suggest that the classic cubic CGL should describe whirling hexagons very well in the supercritical case $A$.

In conclusion, we have numerically investigated whirling hexagons that arise in rotating NB convection and have identified two different regimes. In the weakly NB case the oscillations typically exhibit defect chaos and our analysis suggests that this state should be well described by the 
(a)

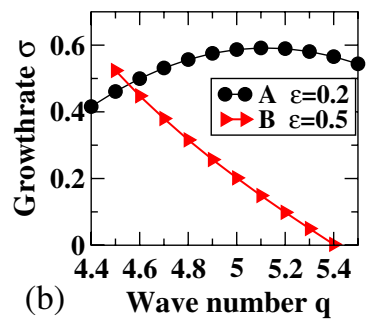

FIG. 6 (color online). (a) $\nabla \cdot \vec{\phi}$ generated by NS burst shown in Fig. 3(b). (b) Growth rate $\sigma$ of the oscillatory mode as a function of the hexagon wave number $q=q_{c}+\nabla \cdot \overrightarrow{\boldsymbol{\phi}} / 2$.

cubic CGL. Rotating NB convection would then represent one of only a few experimentally realizable physical systems [4] in which at least one of the complex states of the two-dimensional CGL is accessible.

For stronger NB effects we found that the Hopf bifurcation becomes subcritical and typical states exhibit bursts. Such bursts and the related retracting fronts have been discussed in some detail previously in one dimension [14-19]. The intermittent bursting behavior, however, has not been studied in detail yet. In two dimensions even less is known. For instance, the conditions for the persistence of bursting when stable steady and whirling hexagons compete remain to be understood. Preliminary simulations indicate that for $\epsilon=0.7$ the bursting persists but not for $\epsilon=0.9$ [cf. Fig. 3(a)].

Since the oscillations arise in a secondary bifurcation the oscillatory mode is, in principle, coupled to the deformations of the hexagon lattice. While in the supercritical case our results indicate that this effect should be small, it is significant in the subcritical case, where deformations of the lattice modify the growth rate of the oscillatory mode substantially [cf. Fig. 6(b)]. Compared to the bursting behavior of the quintic CGL alone, this enriches the scenario and warrants further investigations.

In most of our simulations we have employed periodic boundary conditions and have taken great care to obtain defect-free hexagonal lattices. We have also performed runs in which a radial step in the Rayleigh number to $R=$ 0 mimics a circular container. Defects then tend to destroy the hexagonal lattice. The resulting complex states in which the defects interact with the whirling mode warrant further analysis [cf. [10]]. By stabilizing the hexagon pattern near the boundary through an additional, patterned volume heating we were, however, able to recover the regular lattice and the two chaotic whirling states discussed in the present Letter.

While our numerical experiments have been very productive in identifying and exploring various complex states in rotating NB convection, current computational limitations do not allow us to use the NS simulations to investigate in detail the statistical properties of these states. For instance, for the defect chaos we cannot address the possibility of deviations from the squared Poisson distribution for the defect statistics [22] or the expected transition to exceedingly slow, glassy states [23] as the wave number of the underlying hexagon pattern is changed [9]. Similarly, investigations of the ramifications of the hexagonal anisotropy due to the underlying lattice or of the broken chiral symmetry may require system sizes that are still beyond current computational capabilities. Questions like these can so far only be investigated in experiments.

W.P. and H.R. acknowledge years of stimulating discussions with the late Lorenz Kramer. We gratefully acknowledge support from the Department of Energy (DEFG02-92ER14303).

[1] H. Chaté and P. Manneville, Physica (Amsterdam) 224A, 348 (1996).

[2] I. S. Aranson and L. Kramer, Rev. Mod. Phys. 74, 99 (2002).

[3] J. Burguete, H. Chaté, F. Daviaud, and N. Mukolobwiez, Phys. Rev. Lett. 82, 3252 (1999).

[4] Q. Ouyang and J. M. Flesselles, Nature (London) 379, 143 (1996).

[5] E. Bodenschatz, W. Pesch, and G. Ahlers, Annu. Rev. Fluid Mech. 32, 709 (2000).

[6] F. H. Busse, J. Fluid Mech. 30, 625 (1967).

[7] J.W. Swift, in Contemporary Mathematics (American Mathematical Society, Providence, 1984), Vol. 28, p. 435.

[8] A. M. Soward, Physica (Amsterdam) 14D, 227 (1985).

[9] B. Echebarria and H. Riecke, Phys. Rev. Lett. 84, 4838 (2000); Physica (Amsterdam) 143D, 187 (2000).

[10] Y.-N. Young, H. Riecke, and W. Pesch, New J. Phys. 5, 135 (2003).

[11] All simulations use 6 vertical modes, a time step $\Delta t \leq$ $0.002 h^{2} / \nu_{0}$, and $N^{2}$ horizontal modes ( $N$ as indicated).

[12] The NB coefficients [cf. [10]] at threshold are given by $\gamma_{0}^{(c)}=0.0009, \quad \gamma_{1}^{(c)}=0.2937, \quad \gamma_{2}^{(c)}=-0.1681, \quad \gamma_{3}^{(c)}=$ $0.0215, \gamma_{4}^{(c)}=-0.0022$ for case $A$ and $\gamma_{0}^{(c)}=0.0010$, $\gamma_{1}^{(c)}=0.4885, \quad \gamma_{2}^{(c)}=-0.2281, \quad \gamma_{3}^{(c)}=0.0287, \quad \gamma_{4}^{(c)}=$ -0.0032 for case $B$. The dominant NB effect is the quadratic temperature dependence of the density $\left(\gamma_{1}\right)$.

[13] S. Madruga, H. Riecke, and W. Pesch, J. Fluid Mech. 548, 341 (2006).

[14] L. M. Hocking and K. Stewartson, Proc. R. Soc. A 326, 289 (1972).

[15] P. Kolodner, J. Glazier, and H. Williams, Phys. Rev. Lett. 65, 1579 (1990).

[16] E. Kaplan, E. Kuznetsov, and V. Steinberg, Phys. Rev. E 50, 3712 (1994).

[17] C. S. Bretherton and E. A. Spiegel, Phys. Lett. 96, 152 (1983).

[18] W. Schöpf and L. Kramer, Phys. Rev. Lett. 66, 2316 (1991).

[19] P. Coullet and L. Kramer, Chaos 14, 244 (2004).

[20] M. Sano, H. Kokubo, B. Janiaud, and K. Sato, Prog. Theor. Phys. 90, 1 (1993).

[21] K.E. Daniels, R. J. Wiener, and E. Bodenschatz, Phys. Rev. Lett. 91, 114501 (2003).

[22] C. Huepe, H. Riecke, K. E. Daniels, and E. Bodenschatz, Chaos 14, 864 (2004).

[23] C. Brito, I. S. Aranson, and H. Chaté, Phys. Rev. Lett. 90, 068301 (2003). 